\title{
IMPLANTE DE BALONES PARAURETRALES COMO TRATAMIENTO DE LA INCONTINENCIA URINARIA MASCULINA. EXPERIENCIA DEL HOSPITAL UNIVERSITARIO LA PAZ.
}

José Ramón Cansino Alcaide, Mario Álvarez Maestro, Mario Martín Hernández, Pedro Manuel Cabrera Castillo, Manuel Pérez-Utrilla Pérez, Fermín Rodríguez de Bethencourt, Luís Hidalgo Togores y Jesús Javier De la Peña Barthel.

Servicio de Urología. Hospital Universitario La Paz. Madrid. España.

Resumen.- OBJETIVO: Queremos expresar nuestra experiencia con el implante de balones parauretrales, como tratamiento de la incontinencia urinaria masculina.

MÉTODOS: Hemos revisado de forma retrospectiva nuestra serie desde marzo del 2003 a marzo del 2007, que incluye a 69 pacientes con incontinencia urinaria masculina, la mayoría tras prostatectomía radical.

RESULTADOS: 6 pacientes están pendientes de su primera revisión tras la cirugía. Con un seguimiento medio de 22 meses (3-48) el 57.14\% de los pacientes (36/63) no precisan protección y el $12.69 \%$ usan una compresa de seguridad (8/63), por tanto el 69.83\% (44/63) de los pacientes están secos o usan una compresa de seguridad. Si estratificamos a los pacientes según el grado de su incontinencia, el 81,25\% de los pacientes con incontinencia leve están secos y el $59.25 \%$ de los pacientes en el grupo de incontinencia moderada. Sin embargo, solo el 35\% de los pacientes con incontinencia severa están secos (sin protección).

CONCLUSIÓN: Según nuestra experiencia, pensamos que el implante de balones parauretrales podría ser la primera opción terapéutica para la incontinencia urinaria masculina leve y moderada.

Palabras clave: Incontinencia urinaria. Cirugía prostática.

Summary.- OBJECTIVES: We want to show our experience with paraurethral balloon implantation in the treatment of male urinary incontinence.

METHODS: We retrospectively reviewed our series from March 2003 to March 2007, including 69 male patients with urinary incontinence, most of them after radical prostatectomy.

RESULTS: 6 patients did not have their first follow-up visit after surgery. Mean follow-up was 22 months (3-48). $57.14 \%$ of the patients $136 / 63$ do not need pads, and $12.69 \%$ use one safety pad (8/63); therefore $69.83 \%$ $(44 / 63)$ of the patients are dry or use one safety pad. If we stratify patients by incontinence severity, $81.25 \%$ of the patients with mild incontinence and $59.25 \%$ with moderate incontinence are dry. Nevertheless, only 35\% 
of the patients with severe incontinence are dry (no protection).

CONCLUSIONS: In our experience, we believe that paraurethral balloon implantation could be the first therapeutic option for mild and moderate male urinary incontinence.

Keywords: Urinary incontinence. Prostatic surgery.

\section{INTRODUCCIÓN}

La elección del tratamiento adecuado para el cáncer de próstata localizado sigue resultando una controversia en la actualidad. Para los varones que eligen un tratamiento activo para su neoplasia, la prostatectomía radical (PR) parece ser el método que ofrece mejores resultados en cuanto a control del cáncer a largo plazo (1). Sin embargo este procedimiento no está exento de morbilidad destacando, por su impacto en la calidad de vida del paciente, la Incontinencia Urinaria (IU). Las tasas de IU tras la PR registradas ofrecen unos datos muy variables $13 \%$ $60 \%$ ) (2); estas diferencias se deben a la variabilidad tanto en la definición de la incontinencia como del análisis de la población y de la metodología de investigación.

La IU tras la prostatectomía radical suele ser secundaria a incompetencia intrínseca del esfínter, aunque algunos de estos pacientes presentan una hiperactividad del detrusor concomitante. Las opciones terapéuticas aceptadas incluyen los anticolinérgicos, la rehabilitación perineal, las inyecciones periuretrales de material exógeno, los cabestrillos y la implantación de esfínteres artificiales. Esta última opción ha sido definida por la OMS como técnica ideal en el manejo de la IU tras prostatectomía radical (3).

Desde 1999 Hübner y cols. adaptaron para la IU post-PR la técnica de balones ajustables, que previamente había sido desarrollada para el tratamiento de la IU femenina. Dicha técnica, denominada ProACT (Adjustable Continence Therapy for the prostate) consiste en la implantación percutánea de unos balones de silicona en el espacio parauretral, junto al cuello vesical. Su efectividad se basa fundamentalmente en que permite ajustar de una manera sencilla la presión que necesita cada paciente para estar continente y poder reaccionar además, ante cambios en la presión del detrusor, retención de orina, o alteraciones del suelo pélvico a lo largo del tiempo en caso de necesidad (4).
El objetivo del presente trabajo ha sido revisar, de manera retrospectiva, la eficacia y complicaciones de la técnica ProACT en el tratamiento de la incontinencia urinaria masculina, así como intentar definir su indicación en función de los distintos grados de IU.

\section{MATERIAL Y MÉTODOS}

Desde marzo de 2003 a marzo de 2007, hemos implantado la prótesis ProACT en nuestro servicio a un total de 69 pacientes diagnosticados de incontinencia urinaria refractaria a manejo conservador, la mayoría tras la práctica de PR (60) por cáncer de próstata localizado, 8 tras RTU-P y uno tras traumatismo perineal severo. Aunque hemos implantado la prótesis Pro-Act en un total de 78 pacientes añadiendo los operados en colaboración con otros hospitales, solo hemos incluido en ésta revisión a los pacientes operados y seguidos en nuestro centro. La media de edad fue de 69.82 años (54-78). Todos los pacientes tienen al menos un año de seguimiento y buen control oncológico de su enfermedad (PSA<0.01 ng/ml ó PSA sin modificación en aquellos pacientes con PSA detectable postoperatorio).

El estudio preoperatorio incluyó la anamnesis y categorización de la IU en 3 grupos diferenciados, en función del número de compresas usadas en 24 horas: GRUPO 1 (1-2 compresas) GRUPO 2 (3-4 compresas) GRUPO 3 (>4 compresas). A efectos de valoración de resultados hemos considerado la continencia en sentido estricto como ausencia total de protección. Se completó el estudio con la valoración del tracto urinario mediante cistouretrografía miccional, flujometría y urocultivo para descartar obstrucción a nivel de la anastomosis y/o infección urinaria. Realizamos uretrocistoscopia como paso inicial al procedimiento de implante de ProACT. La práctica de estudio urodinámico ha quedado reservada en la actualidad a los pacientes con sospecha de vejiga inestable.

Realizamos una revisión retrospectiva de la serie aportando los resultados obtenidos mediante la revisión de las historias clínicas. La totalidad de los implantes corrió a cargo de dos cirujanos (L.H.T. y R.C.A.)

\section{Técnica quirúrgica}

La técnica quirúrgica es relativamente sencilla y de corta duración (aproximadamente $30 \mathrm{~min}$.). Bajo anestesia regional, se coloca al paciente en posición de litotomía con la mesa paralela al suelo del quirófano. Tras la realización de uretrocistoscopia, 


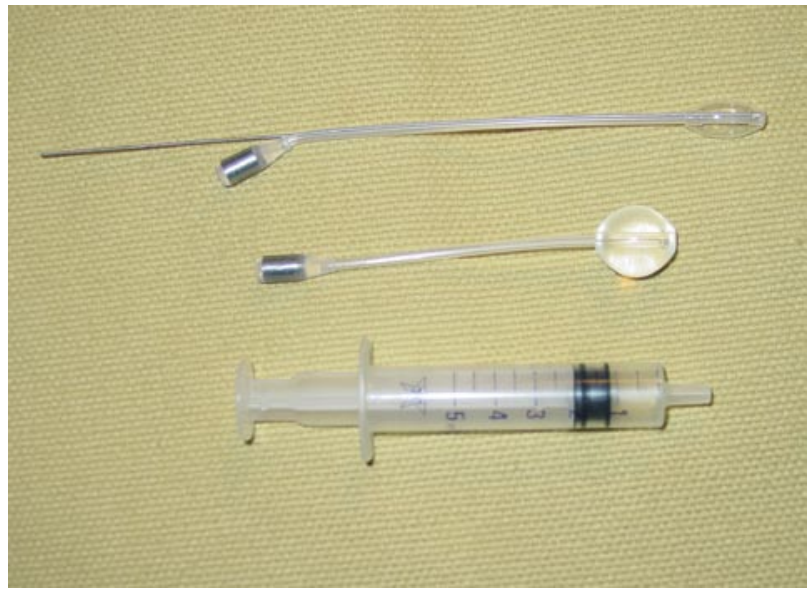

FIGURA 1. Próstesis PRO-ACT: 2 balones regulables.

se rellena la vejiga con contraste para efectuar una cistografía con el arco en " $\mathrm{C}$ " a $90^{\circ}$ respecto a la mesa del quirófano.

Mediante una incisión perineal de aproximadamente $2.5 \mathrm{cms}$. y con ayuda de unas pinzas romas tipo Crawford, desarrollamos un trayecto paralelo al cistoscopio, atravesando el diafragma urogenital y controlando de forma fluoroscópica la localización del extremo de la pinza inmediatamente distal y lateral al cuello vesical, sin dañar la uretra ni la vejiga (control endoscópico y fluoroscópico simultáneo) (Figuras 1 y 2 ).

En caso de perforación con fuga de contraste observado fluoroscópicamente o con visión directa del cistoscopio, se abandonará ese lado para realizarse en otro acto quirúrgico posteriormente, entre 6 semanas y 3 meses después. Una vez desarrollado el trayecto transperineal, se coloca un trócar especial como vaina introductora previo a la retirada del Crawford, a través de la cual se inserta el balón vacío. El balón tiene un alambre guía que aporta consistencia a la prótesis y una morfología helicoidal vaciado que facilita su deslizamiento y su correcta ubicación. Bajo control fluoroscópico se aloja en la localización elegida sobre la vaina introductora. Seguidamente se rellena el balón con aproximadamente $2 \mathrm{ml}$. de una mezcla isotónica de contraste y agua estéril. Ésta maniobra, realizada bajo control fluoroscópico, permite controlar el llenado del balón tras lo cual retiraremos la vaina introductora y el alambre guía. Con la visión endoscópica observamos la protrusión del dispositivo sobre la uretra. Aprovechando la incisión perineal, desarrollamos un trayecto subcutáneo en el escroto, donde quedará alojada la válvula o conexión de titanio para posteriores ajustes. El cierre de la incisión se realiza mediante sutura reabsorbible de 4/0.

Los pacientes son dados de alta incontinentes y con escaso relleno de los balones para evitar retenciones de orina. El objetivo es conseguir una correcta fibrosis alrededor de los balones durante 6 semanas, periodo durante el cual no realizaremos rellenos para evitar el desplazamiento de los mismos.

El relleno se realiza de forma ambulante y $\sin$ necesidad de anestesia. Se localiza la conexión de titanio alojada bajo la piel escrotal por palpación y con aguja de insulina y jeringa cargada con una mezcla de agua estéril y contraste idéntica a la empleada durante la cirugía, se procede al relleno. Es preferible un mayor número de rellenos con escaso volumen, que menos rellenos con mayor volumen corriendo alto riesgo de entrar en retención aguda de orina (Figuras 3 y 4 ).
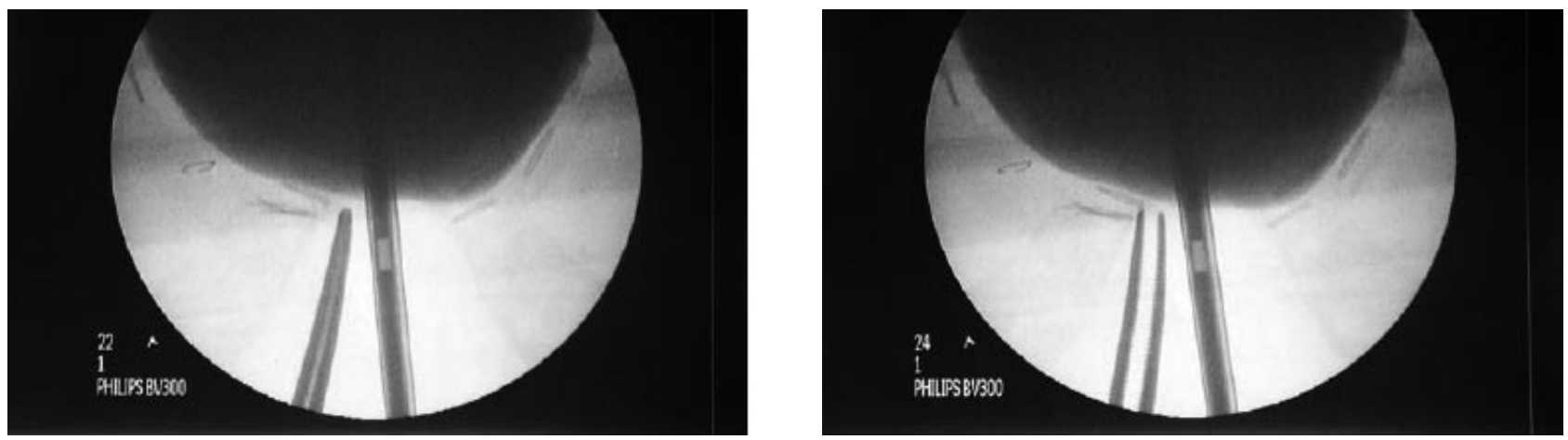

FIGURA 2. Incontinencia post-cirugía prostática: implante de prótesis PRO-ACT. 
En caso de necesidad, la retirada de los balones resulta bastante sencilla y puede realizarse en régimen ambulatorio: La conexión de titanio alojada bajo la piel, a nivel escrotal se localiza con facilidad por palpación. Bajo anestesia local, se realiza una pequeña incisión de 0.5-1 cm. para extraer dicha conexión. Se vacía el balón de su contenido, mediante punción con aguja y jeringa, retirando el dispositivo por tracción, habitualmente sin dificultad (Figuras 5 y 6$)$

\section{RESULTADOS}

El seguimiento medio fue de 22 meses (3$48)$. Doce pacientes habían sido sometidos previamente a otros tratamientos para el control de la IU: 12 pacientes a inyección periuretral de colágeno y 2 pacientes tras retirada de esfínter artificial (estos casos habían recibido previamente inyección periuretral de colágeno).

Seis pacientes del total (6/69), que están pendientes de revisión en consulta no han sido incluidos en el análisis de los resultados.

El $57.14 \%$ de los pacientes $(36 / 63)$ no precisan protección y el $12.69 \%$ usan una compresa de seguridad (8/63), por tanto el 69.83\% (44/63) de los pacientes están secos o usan una compresa de seguridad. El 14.28\% (9/63) han notado una mejoría objetiva al disminuir el número de compresas que usan en la actualidad, en comparación con su situación preoperatoria. Diez pacientes no refieren ninguna mejoría (15.87\%) y de ellos a $7 / 10$ se les ha implantado un esfínter artificial con buenos resultados en la actualidad.

Cuando estratificamos según la gravedad de la IU, encontramos 16 pacientes en el grupo 1, a 27 pacientes en el grupo 2 y 20 pacientes en el grupo 3. El análisis de resultados por grupos nos muestra que dentro del grupo 1, 13/16 pacientes no precisan ningún tipo de protección tras la colocación del ProACT, lo que constituye un $81.25 \%$ y si sumamos a un paciente más que usa una compresa de seguridad, la mejoría se eleva al $87.50 \%$ (14/16). En el grupo $2 ; 16 / 27$ pacientes no precisan protección $(59.25 \%)$ ascendiendo hasta un $74.06 \%$ (20/27) si sumamos a 4 pacientes que usan una compresa de seguridad. En el grupo $3 ; 7 / 20(35 \%)$ pacientes consiguen una continencia completa, que asciende a un $50 \%(10 / 20)$ contando con los pacientes que usan una compresa de seguridad. La mejoría global (pacientes que se encuentran mejor tras el implante de los balones usando menos protección) fue del $85 \%$. (17/20) (Figuras 7 y 8$)$.
La media de rellenos es de 1.9 por paciente (26 pacientes con 2 rellenos) y el volumen medio por cada balón es de 3.06 .

\section{Entre las complicaciones destacan}

4/69 casos $(5.79 \%)$ presentaron retención aguda de orina resueltos mediante sondaje vesical y posterior vaciado de los balones, sin alterar el buen funcionamiento posterior de la prótesis. 7/69 (10.14\%) pacientes (10 perforaciones) presentaron perforación uretra o vejiga durante el implante. En estos casos se realizó implante diferido 16 semanas a 3 meses) sin diferencias significativas en cuanto a resultados. $1 / 69$ (1.44\%) presentó salida del balón por herida perineal en un paciente, en relación a infección con erosión local. 2/69 (2.88\%) pacientes expulsaron un balón por el recto manejado de manera conservadora. 1/69 (1.44\%) un paciente diabético presentó una infección de la prótesis, resuelta con retirada de la misma y antibioterapia específica. $2 / 69(2.88 \%)$ sufrieron estallido de un balón durante un relleno (secundario a defecto en la primera generación de balones, actualmente retirada). $2 / 69(2.88 \%)$ precisaron retirada de un balón para su recolocación por mala ubicación consiguiendo mejorar el funcionamiento de la prótesis en uno de ellos. 4/69 (5.79\%) pacientes son portadores de un solo balón de los cuales 3 están continentes sin necesidad de protección; en el caso restante carecemos de información por estar pendiente de revisión clínica.

\section{DISCUSIÓN}

La incontinencia urinaria resulta una complicación significativa tras la prostatectomía radical y supone un deterioro manifiesto de la calidad de vida en los pacientes que la padecen. De hecho la preocupación en el momento del diagnóstico de cáncer de próstata por la IU resulta de mayor impacto que la disfunción eréctil y es motivo de cambios en la indicación terapéutica por parte del paciente. Aunque, como consecuencia de la mejoría en la técnica quirúrgica, se ha registrado una disminución en la tasa de incontinencia a lo largo de la última década, el número de pacientes con IU tras cirugía prostática se ha incrementado de manera paralela al número de procedimientos anualmente realizados.

La continencia tras la prostatectomía radical depende de múltiples factores. Así encontramos factores preoperatorios (edad, tabaquismo, comorbilidad neurológica, resección transuretral de próstata previa), factores predictivos intraoperatorios (estadio, anatomía periprostática, tensión de la anastomosis) 


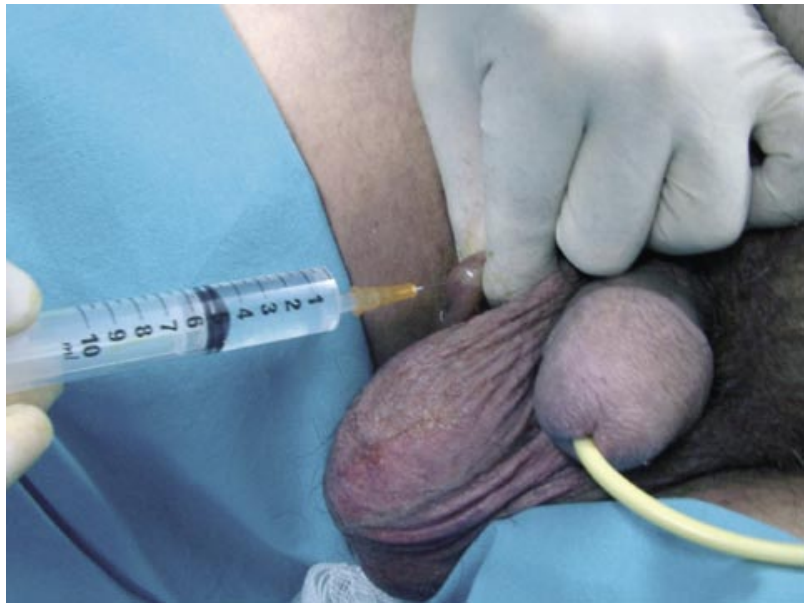

FIGURA 3. Relleno regulable ambulatoriamente.

y factores postoperatorios (fuga urinaria, hematoma postoperatorio) (5). Aunque, como hemos mencionado previamente, la curva de aprendizaje y el adecuado seguimiento postoperatorio pueden ayudar a mejorar los resultados en cuanto a continencia, resulta evidente que muchos de estos no son susceptibles de modificación.

Siguiendo el criterio de la OMS, el "gold standard" en el tratamiento de la IU post-prostatectomía es el implante de esfínter artificial; sin embargo, hay autores que lo cuestionan, ya que éste procedimiento no está exento de complicaciones, entre las cuales destacan la erosión uretral, la persistencia de la incontinencia y una tasa no desdeñable de reintervenciones para retirada del dispositivo por infección o malfuncionamiento $(2,12)$.

Se han intentado distintos procedimientos que conlleven unos menores costes económicos o asociados a procedimientos mínimamente invasivos. Entre ellas la utilización de medicación oral anticolinérgica, la rehabilitación perineal, las inyecciones periuretrales de material exógeno y los cabestrillos. El resultado global de estos procedimientos ha sido variable $y$, con frecuencia, poco satisfactorio en el seguimiento a largo plazo (3).

El diseño de la Prostate Adjustable Continence Therapy (ProACT; Uromedica, Plymouth, Minnesotta) supone el implante de dos balones para-uretrales, uno a cada lado de la uretra, junto al cuello vesical, con el objetivo de incrementar la resistencia uretral. Lo que diferencia a este dispositivo de otros procedimientos en la terapia para la IU es la presencia de dos conexiones conectadas a los balones, que permiten ajustes postoperatorios del volumen de los balones mediante inyección percutánea (4).

La valoración de las tasas de continencia tras la prostatectomía radical resulta difícil puesto que la metodología y la selección de pacientes no son comparables en las distintas series estudiadas. No existe un criterio uniforme en la definición de continencia postoperatoria; mientras unos autores consideran continente exclusivamente a pacientes que no utilizan protección, otros grupos admiten el uso de una compresa diaria como protección sin alterar el criterio de continencia. Utilizando criterios estrictos la tasa de continencia trancurrido un año de la prostatectomía radical laparoscópica resulta de un $84 \%$ (6). Esa tasa resulta similar a la descrita en las series contemporáneas de prostatectomía radical retropúbica $(80-95 \%)(7,8)$.

La predicción del tiempo hasta obtener la continencia es un factor determinante a la hora de manejar ésta. En los primeros tres meses tras la cirugía, hasta un $24 \%$ de los pacientes presentan IU en
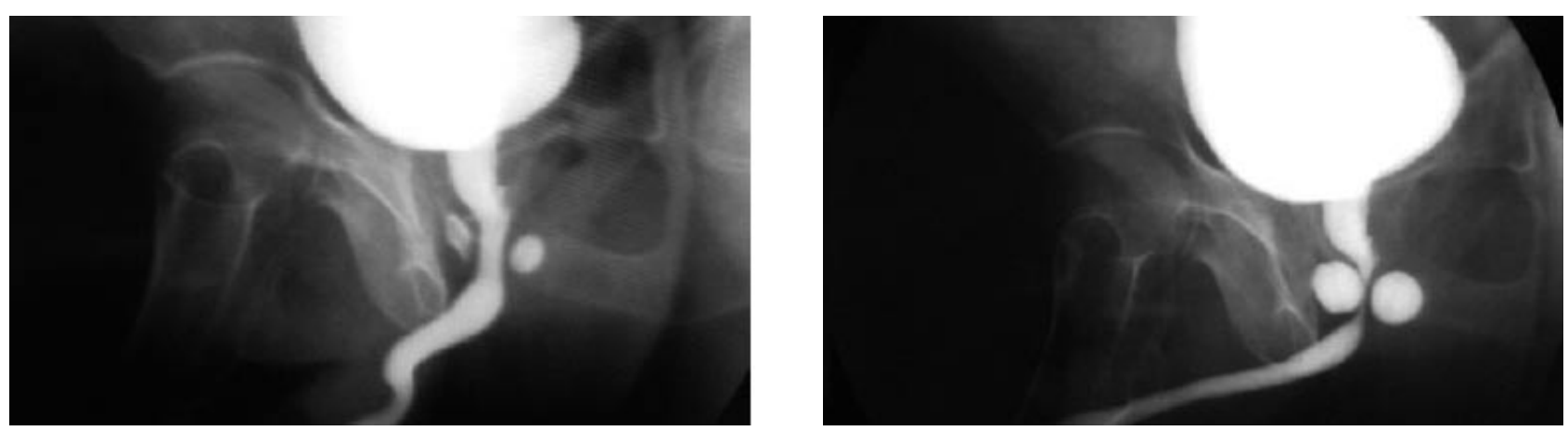

FIGURA 4. Incontinencia post-cirugía prostática: implante de prótesis PRO-ACT. Relleno de balones. $+1,5$ cc en cada balón. 


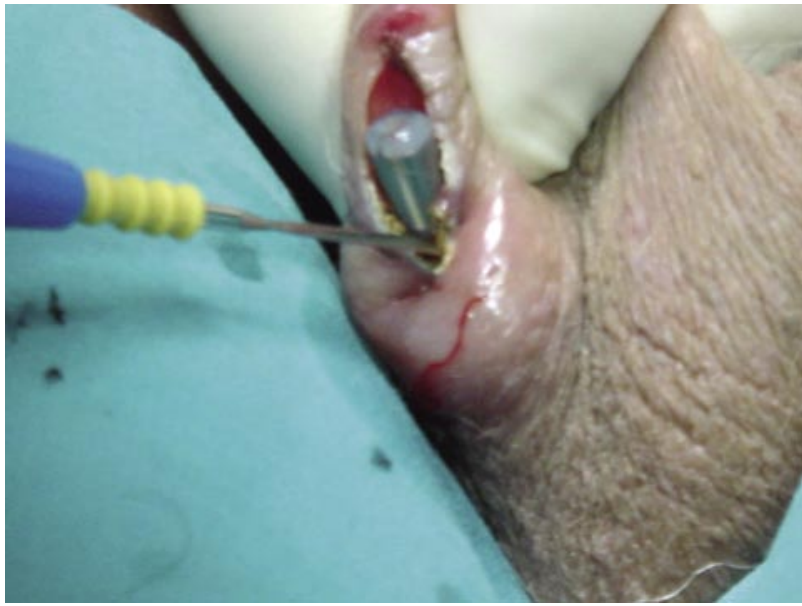

FIGURA 5. Infección implante derecho: retirada con anestesia local.

distinto grado; en esta fase los ejercicios de rehabilitación perineal pueden contribuir al restablecimiento del control esfinteriano (9). Aunque pasados seis meses las posibilidades de corrección disminuyen considerablemente se establece que el tiempo de recuperación espontánea a continencia total es de un año, aunque puede extenderse hasta los dos años (10). Pese a que la valoración de algunos factores, arriba mencionados, como la edad, la técnica quirúrgica o las complicaciones postoperatorias, pueden ayudarnos a predecir la continencia, no disponemos de ningún modelo preoperatorio eficaz para la predicción de esta.

Recientemente Ates y cols. (1 1) han descrito un modelo predictivo de continencia postoperatoria,

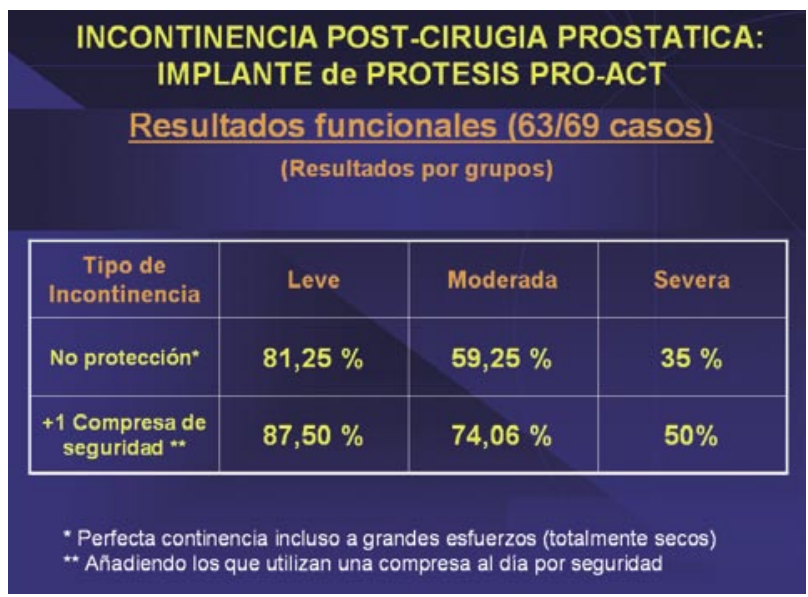

FIGURA 7

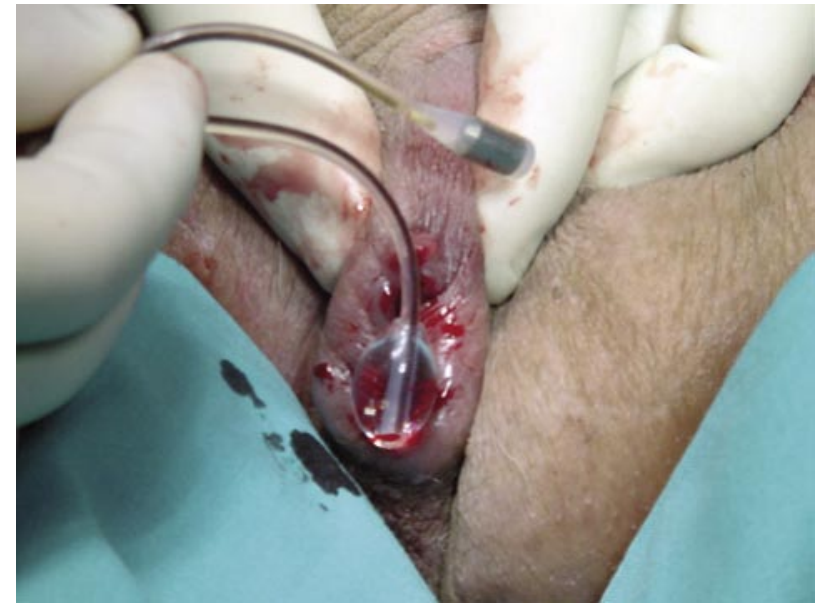

FIGURA 6. Infección implante derecho: retirada con anestesia local.

basándose en el volumen de pérdida de orina en relación con el volumen total de diuresis diaria realizan una predicción del tiempo que transcurre hasta obtener una continencia completa. Pérdidas urinaria por encima del $15 \%$ del volumen de diuresis diaria suponen un alto riesgo de incontinencia.

En nuestra serie hemos incluido pacientes con IU de al menos un año de evolución, puesto que en nuestra experiencia (datos no publicados) no encontramos mejoría significativa del síntoma incontinencia, transcurridos los doce primeros meses. En todos los casos, buscando una pronta recuperación de la IU, se recomendó a los pacientes la realización de maniobras de Kegel y en algunos casos, se administró tratamiento antimuscarínico complementario. Aquellos casos en los que predominó el síntoma urgencia-incontinencia recibieron tratamiento médico tras estudio urodinámico, aunque algunos autores refieren una buena respuesta clínica tras el incremento a la resistencia uretral tras cirugía en ambos grupos (12). No hemos incluido entre nuestras opciones terapéuticas algunas opciones conservadoras como el bioffedback ultrasónico transrectal o la electroestimulación; éstas pueden resultar una alternativa válida en el manejo de la IU tras cirugía prostática, especialmente en las fases iniciales y en las IU de bajo débito.

Para el análisis de la muestra hemos definido como pacientes continentes a aquellos que no utilizan protección, aquellos que utilizan 1-2 compresas se consideran incontinencia leve (grupo 1), moderada (grupo 2) de 3-4 compresas y grave (grupo 3) a aquellos que utilizan más de 4 compresas por día. Este criterio es objeto de debate; algunos autores cla- 


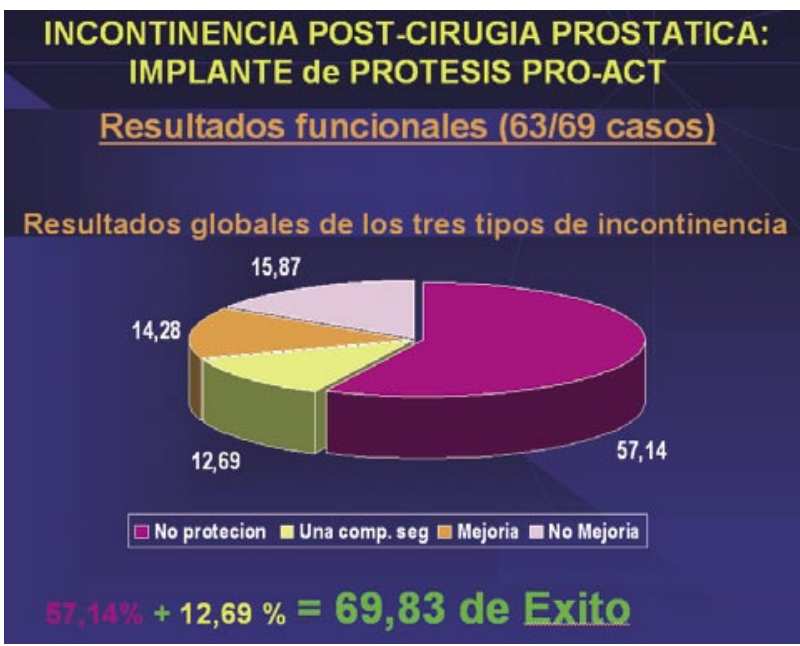

FIGURA 8

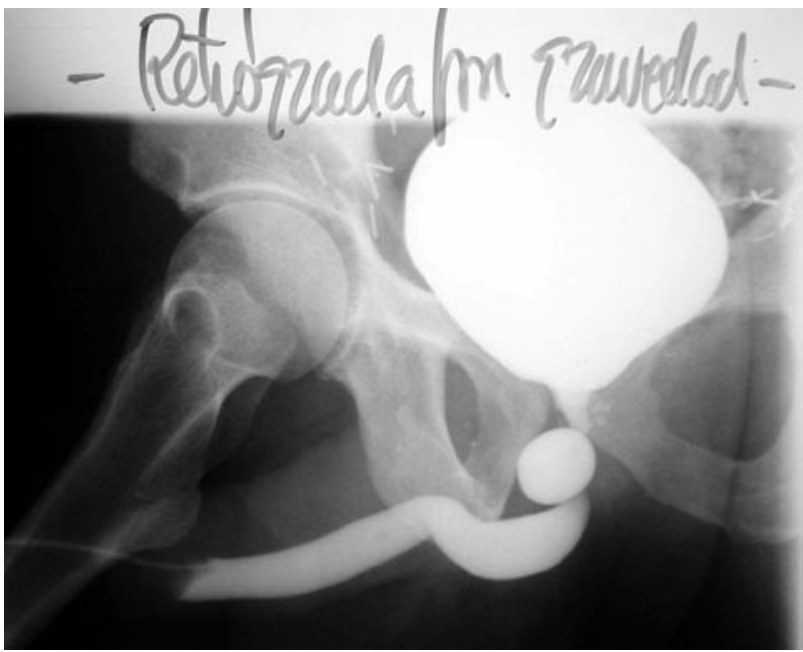

FIGURA 9. Uretrocistografía retrógrada con implante de balón único. sifican como pacientes continentes a aquellos que utilizan 0-1 compresas por día 12; sin embargo, dada la incidencia en la calidad de vida de la IU, hemos considerado en nuestro grupo como pacientes continentes a aquellos que pueden desarrollar una vida normal y eso incluye la no utilización de protección. Hemos obtenido en nuestra experiencia unos buenos resultados con una continencia (ausencia de protección) de 57.14 \% de forma global; sumando los pacientes continentes con los que utilizan una compresa de protección obtenemos un $69.83 \%$; la tasa de mejoría global (ausencia de protección ó disminución en el número de compresas diarias) asciende hasta el $84.08 \%$. Cuando analizamos los resultados de conti-

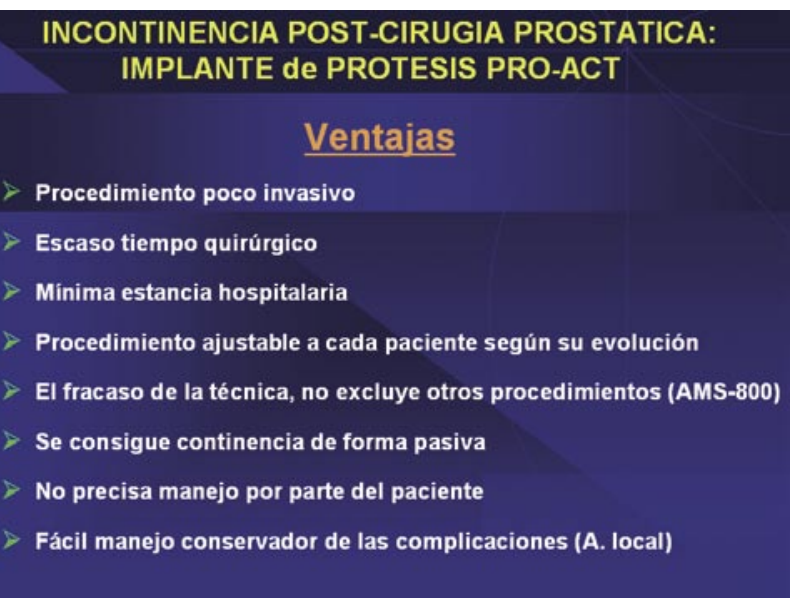

FIGURA 10. nencia en función de los grupos de incontinencia arriba definidos, encontramos unas tasas de continencia para los grupos 1,2 y 3 de $81.2 \%, 59.2 \%$ y $35 \%$ y sumando a los pacientes que usan una compresa de seguridad; $87.5 \%, 74 \%$ y $50 \%$. En nuestro criterio, la baja tasa de continencia completa en el grupo 3, supone que debamos cuestionarnos la indicación del implante de ProACT en éstos pacientes, en contra de lo que opinan Hübner y Trigo-Rocha $(4,12)$.

El estudio preoperatorio de los pacientes ha incluido la valoración del tracto urinario superior mediante ecografía, del tracto urinario inferior mediante uretrocistografía retrógrada y hemos descartado la existencia de obstrucción mediante flujometría. En todos los casos se descartó la coexistencia de infección urinaria, que por lo demás es infrecuente en estos pacientes, habida cuenta de la ausencia de residuo postmiccional. Realizamos uretroscopia como paso inicial al procedimiento de implante de ProACT; no consideramos necesario incluir este medio diagnóstico en la batería preoperatoria, puesto que en ninguno de los casos estudiados, utilizando la pauta arriba descrita, hemos encontrado anomalías que supusieran modificación o aplazamiento del procedimiento. La práctica de estudio urodinámico ha quedado reservada a los pacientes con sospecha de vejiga inestable. En todos los casos ha resultado condición indispensable el adecuado control del tumor; para ello se exigió niveles de PSA $<0.01 \mathrm{ng} / \mathrm{ml}$ durante un periodo de un año; aquellos pacientes que presentaron niveles detectables de PSA postoperatorio mantuvieron sus cifras invariables durante un periodo superior a un año. Para los pacientes de alto riesgo de recurrencia local o sistémica (PSA preoperatorio $>10 \mathrm{ng} / \mathrm{ml}$, estadios avanzados o grado histológico 
alto), se recomienda un seguimiento superior a 18 meses sin evidencia de recurrencia.

El procedimiento quirúrgico resulta sencillo y no precisa de apoyo tecnológico especial. Tan sólo resulta necesaria cierta familiaridad combinando endourología y fluoroscopia, habitual en nuestra especialidad para el manejo endourológico del tracto urinario superior. Por ello el aprendizaje ha resultado sencillo y la tasa de complicaciones ha sido baja y en su mayoría de escasa entidad. En aquellos casos en los que exista alguna dificultad técnica para la utilización del "arco en C" o ante la preferencia del cirujano, puede realizarse el implante bajo control de ecografía transrectal que evitaría las perforaciones rectales, aunque nosotros preferimos el control radiológico que nos ayuda en el seguimiento postoperatorio y nos permite tomar una decisión acertada a la hora de realizar los rellenos (13).

La complicación más frecuente ha sido la perforación vesical o uretral (7/69 pacientes, 10.1\%) Esta complicación resultó más frecuente en la fase inicial. La radioterapia previa es contraindicación para este procedimiento, ya que incrementa el riesgo de fracaso y de complicaciones, como consecuencia de la fibrosis actínica inducida. En los casos de perforación vesical o uretral el manejo ha sido expectante, con retirada del dispositivo, sondaje vesical y aplazamiento en el implante (6 semanas); sin embargo esta complicación no implica la anulación del procedimiento.

La segunda complicación en frecuencia ha resultado la retención aguda de orina $(4 / 69,5.79 \%)$ y ha sido la consecuencia de un aumento en la resistencia uretral secundario a un excesivo llenado de los balones. Su manejo ha sido sencillo, gracias al dispositivo de conexión de los balones de ProACT que permite tanto el llenado como el vaciado de éstos. En todos los casos esta complicación ha sido resuelta de manera ambulante, sin precisar ulterior manipulación en los pacientes y consiguiendo una adecuada micción y buen control de la continencia.

Las infecciones y las disfunciones de los balones han sido manejadas con facilidad, pudiendo retirar los balones afectados mediante una mínima incisión escrotal bajo anestesia local. Resulta llamativo que un $75 \%(3 / 4)$ de los pacientes portadores de un solo balón se encuentran continentes en la actualidad, no habiéndose visto su situación clínica comprometida por el deterioro contralateral ni por la manipulación para la retirada de este (Figura 9). En nuestra serie encontramos dos casos de perforación espontánea a recto y eliminación transrectal del globo.Uno de ellos tenía antecedente de Braquiterapia prostática y posterior cirugía radical de rescate, aunque ambos se resolvieron de manera espontánea. En el caso descrito por Kempkensteffen y cols. (14) el paciente había sido sometido a radioterapia previa; la fibrosis y la peor situación vascular asociada a este tratamiento adyuvante podría haber facilitado la erosión y posterior perforación rectal. Como en nuestro caso la perforación se resolvió espontáneamente, sin necesidad de otras actuaciones quirúrgicas ni ingreso hospitalario.

La colocación de un esfínter artificial se ha considerado tradicionalmente como el sistema más efectivo para tratar la IU tras cirugía prostática. Otros métodos utilizados han mostrado eficacia en IU (inyección periuretral de sustancias exógenas y colocación de slings uretrales), pero su eficacia en el tiempo o ante incontinencias graves ha resultado menor (3). Sin embargo la colocación de un esfínter externo no está exenta de complicaciones: el fallo mecánico, la erosión uretral y la infección del dispositivo, aunque han disminuido, siguen dando lugar a una tasa de reoperación que se acerca al 17\% (15). Además el implante de un esfínter artificial va asociado a un elevado coste económico que, en los tiempos actuales, no puede ser obviado. La inyección periuretral también va asociado a un elevado coste, habida cuenta de la pérdida de eficacia de éstas a lo largo del tiempo y la necesidad de inyecciones repetidas o tratamientos alternativos.

En relación a los slings, consideramos que el ProACT aporta algunas ventajas como es la preservación uretral y la facilidad de retirada del dispositivo, especialmente en aquellos pacientes en los que una mala respuesta terapéutica suponga una ulterior colocación de esfínter artificial (Figura 10).

\section{CONCLUSIONES}

En nuestra experiencia el implante de balones ProACT para el tratamiento de la incontinencia urinaria tras cirugía prostática, resulta una técnica segura, efectiva y de fácil aprendizaje. Las complicaciones asociadas han registrado un número escaso y su gravedad ha sido baja; el manejo para su solución no ha implicado una morbilidad asociada. La valoración de los resultados tras la colocación de un alto número de dispositivos aporta unos buenos resultados en cuanto a continencia o mejoría de esta, especialmente en los casos que presentan un grado menor de incontinencia postoperatoria.

Para los casos de incontinencia severa creemos que la elección en la actualidad sigue siendo la colocación de un esfínter artificial; sin embargo el 
manejo mediante el sistema ProACT en este grupo de pacientes no compromete la ulterior sustitución por esfínter artificial en aquellos pacientes que lo precisen. Consideramos por lo tanto que en atención a parámetros de coste-efectividad, facilidad de aprendizaje y escasa morbilidad asociada, el implante de balones ProACT resulta una técnica útil en el manejo de la IU leve y moderada tras la cirugía prostática.

Pensamos que es la primera opción para el tratamiento de la incontinencia leve-moderada tras cirugía prostática.

\section{BIBLIOGRAFÍA y LECTURAS RECOMENDADAS ( lectura fundamental)}

1. HOLMBERG, L., BILL-AXELSON, A.; HELGESEN, F. y cols.: "Scandinavian Prostatic Cancer Group Study Number 4: A randomized trial comparing radical prostatectomy with watchful waiting in early prostate cancer". N Engl J Med;347:. 2002.

2. DALKIN, B.L.; WESSELLS, H.; CUI, H.: "A national survey of urinary and health related qualityof life outcomes in men with an artificial urinary sphincter for post-radical prostatectomy incontinence". J. Urol. 169, 237, 2003.

3. HERSCHORN, S.; THUROIFF, J.; BRUSCHINI, H. y cols.: "Surgical treatment of urinary incontinence in men". In: P. Abrahms, L. Cardozo, S. Khoury and A. Wein, Editors, Incontinence, vol. 2. Third International Consultation on Incontinence, Health Publications, Paris, France, p. 1250, 2005.

**4. HÜBNER, W.A. y SCHLARP, O.M.: "Treatment of incontinence after prostatectomy using a new minimally invasive device: adjustable continence therapy". BJU Int 96. 5872005.

5. KOPPIE, T.M.; GUILLONNEAU, B.: "Predictors of Incontinence after Radical Prostatectomy: Where Do We Stand?". Eur. Urol., doi:10.1016/ j.eururo. 2007.
6. KUNDU, S.D.; ROEHL, K.A.; EGGENER, S.E. y cols.: "Potency, continence and complications in 3,477 consecutive radical retropubic prostatectomies". J Urol.; 172: 2227. 2004.

7. JACOBSEN, N.E.; MOOORE, K.N.; ESTEY, E. y cols.: "Open versus laparoscopic radical prostatectomy: A prospective comparison of postoperative urinary incontinence rates". J. Urol 177615. 2007.

**8. EASTHAM, J.A.; KATTAN, M.W.; ROGERS, E. y cols.: "Risk factors for urinary incontinence after radical prostatectomy". J. Urol. 156:1707, 1996.

*9. GEARY, E.S.; DENDINGER, T.E.; FREIHA, F.S. y cols.: "Incontinence and vesical neck strictures following radical retropubic prostatectomy". Urology 45: 1000, 1995.

10. STOLZENBURG, J.U.; LIATSIKOS, E.N.; RABENALT, R. y cols.: "Nerve sparing endoscopic extraperitoneal radical prostatectomy--effect of puboprostatic ligament preservation on early continence and positive margins". Eur Urol. 49:103, 2006.

11. ATES, M.; TEBER, D.; GOZEN, A.S. y cols.: "A new postoperative predictor of time to urinary continence after laparoscopic radical prostatectomy: the urine loss ratio". Eur. Urol.; doi:10.1016/ j.eururo.2006.12.022. 2007.

**12. TRIGO-ROCHA, F.; MENDES GOMES, C.; LIMA POMPEO, A.C. y cols.: "Prospective study evaluating efficacy and safety of Adjustable Continence Therapy (ProACT) for post radical prostatectomy urinary incontinence". Urology 67: 965, 2006.

*13. GREGORI, A.; SIMONATO, A.; LISSIANI, A. y cols.: "Transrectal ultrasound guided implantation of the ProACT adjustable continence therapy system in patients with post-radical prostatectomy stress urinary incontinence: A Pilot Study". J. Urol. 176: 2109, 2006.

*14. KEMPKENSTEFFEN, C.; HINZ, S.; CHRISTOPH, F. y cols.: "Rectal perforation as a late complication of ProACT implantation". Urologe A. 45:865, 2006.

*15. ELLIOTT, D.S.; BARRETT, D.M. "Mayo Clinic long-term analysis of the functional durability of the AMS 800 artificial urinary sphincter: a review of 323 cases". J Urol.;159:1206, 1998. 